\title{
Self-similar drag reduction in plug-flow of suspensions of macroscopic fibers
}

\author{
J. J. J. Gillissen ${ }^{1}$ and J. P. Hoving ${ }^{2}$ \\ ${ }^{1}$ Department of Chemical Engineering, Delft University of Technology, Julianalaan 136, \\ 2628 BL Delft, The Netherlands \\ ${ }^{2}$ Fokker Aerostructures, Edisonstraat 1, 7900 AB Hoogeveen, The Netherlands
}

(Received 4 July 2012; accepted 17 October 2012; published online 8 November 2012)

\begin{abstract}
Pipe flow experiments show that turbulent drag reduction in plug-flow of concentrated suspensions of macroscopic fibers is a self-similar function of the wall shear stress over the fiber network yield stress. We model the experimental observations, by assuming a central fiber network plug, whose radius is determined by the yield stress. According to the model the plug constrains the size of the turbulent eddies in the surrounding annulus, with the effect of a reduced friction factor as compared to Newtonian flow. (c) 2012 American Institute of Physics. [http://dx.doi.org/10.1063/1.4766198]
\end{abstract}

In this Letter, we present friction versus flow rate experiments for the pipe flow of suspensions of macroscopic fibers. At large enough concentrations these fibers form a stable network, in the central region of the pipe, confining the remnants of the original turbulent flow to the annular wall region. In this plug-flow regime, the friction factor is reduced as compared to the Newtonian flow. In this work, we examine the scaling of this drag reduction and present a mathematical model that captures the experimental data. According to the model, the rigid network in the core improves the flow, by constraining the size of the turbulent eddies in the annulus.

Drag reduction in pipes, ducts, and channels has received much attention in the literature, and the phenomenon has mostly been studied for polymers, ${ }^{1}$ but also for macroscopic fibers, ${ }^{2}$ gas bubbles, ${ }^{3}$ surfactants ${ }^{4}$ or clay particles. ${ }^{5}$ For a recent overview, see Ref. 6 . Polymers are the most effective, in terms of drag reduction per concentration of the additive. It is known from simulations and experiments that polymer drag reduction scales with $c r_{a}^{2}$, where $c$ is the volumetric polymer concentration, $r_{a}=l / d$ is the polymer aspect ratio, $l$ is the polymer length, and $d$ is the polymer diameter. ${ }^{1,7,8}$ The large aspect ratio of extended polymers $r_{a} \sim 10^{4}-10^{5}$ explains that significant drag reduction can be obtained at very small polymer concentrations $c \sim 10^{-5}-10^{-4}$.

Drag reduction has also been measured using macroscopic fibers, such as wool, wood, nylon or asbestos. ${ }^{2,9}$ The notion that a large aspect ratio is key for drag reduction, suggests that polymers and fibers reduce the drag according to similar mechanisms. The contrary is observed from experiments, however, showing marked differences in the profiles of the mean flow. Polymers act in the nearwall, buffer layer, effectively thickening the viscous sublayer, while unaffecting the momentum transfer in the turbulent core. ${ }^{1}$ Macroscopic fibers, on the other hand, act in the turbulent core, while unaffecting the near-wall region. ${ }^{2,9}$ This difference stems from the different lengths of polymers and fibers. Polymers are usually of sub-Kolmogorov length scale. On the scale of the near-wall vortices, the polymers can be regarded as a continuum that induces an additional viscosity, which dampens the momentum transfer of the near-wall vortices. ${ }^{10}$ Fibers, on the other hand, are usually orders of magnitude larger than the near-wall vortices. Instead of inducing internal friction, the fibers impose external constraints on the near-wall vortices. The presence of the fibers requires the vortices to re-organize.

Measurements of the velocity profiles in fibre-induced, drag-reduced flow show several flow regimes. ${ }^{2}$ At large enough Reynolds numbers or small enough concentrations, the flow is identical to that of the pure solvent. With increasing concentration the central profile slope first increases and then 
decreases, which suggests the following two opposing mechanisms. Freely moving fibers dampen the momentum transferring eddies, while fibre agglomerates enhance the momentum transfer. With a further increase in the fibre concentration, the agglomerates form a network, capable of supporting the shear stress in the fluid. The result is a central fiber network plug with a uniform velocity, surrounded by a turbulent annulus. ${ }^{2,11}$ With increasing fibre concentration the plug radius grows and when the network spans the whole pipe cross section, the pipe is clogged.

The formation of the network plug with physical contacts between the fibers requires the number of contacts between a fiber and its neighbours to be $c r_{a} \gtrsim 1$. Most previous studies on fibre drag reduction are restricted to $c \lesssim 10^{-2}$ and $r_{a} \lesssim 10^{2}$, and therefore the "plug-flow" regime has not yet been systematically explored.

In this Letter, we use round fibers with a relatively large aspect ratio $r_{a}=4 \times 10^{2}$. With this large $r_{a}$, we are able to study the plug-flow regime over a decade of fibre concentrations, revealing new insights in the scaling behaviour of this flow.

We view drag reduction as the increase of the bulk velocity $U_{b}$ at a fixed pressure gradient $d P / d x$. Using this view, it is natural to scale $U_{b}$ with the friction velocity

$$
U_{\tau}=\sqrt{\frac{\tau_{w}}{\rho}}
$$

and to define drag reduction as the increase of the scaled bulk velocity $U_{b}^{+}=U_{b} / U_{\tau}$ at a constant frictional Reynolds number

$$
R e_{\tau}=\frac{U_{\tau} D}{v} .
$$

Here, $\rho$ is the fluid mass density, $v=\mu / \rho$ is the fluid kinematic viscosity, $\mu$ is the fluid dynamic viscosity, $\tau_{w}=(D / 4)(d P / d x)$ is the wall shear stress, and $D$ is the pipe diameter.

Our experimental setup consists of a centrifugal pump that drives the fiber suspension through a loop. The loop contains a $4 \mathrm{~m}$ test section, consisting of a transparent, straight and smooth perspex pipe with an internal diameter of $D=5 \times 10^{-2} \mathrm{~m}$. The pressure drop is measured over the last $2.2 \mathrm{~m}$ of the test section, using membrane differential pressure transducers (Validyne: DP15 and DP45). The flow rate is measured using a magnetic inductive flow meter (Krohne Altometer: IFS 4000). A video camera on a rail is used to visualize the dynamics of the flow in a co-moving frame of reference. We use nylon fibers in tap water. The fibers have a width of $d=1 \times 10^{-5} \mathrm{~m}$ and a length of $l=4 \times 10^{-3} \mathrm{~m}$, corresponding to an aspect ratio of $r_{a}=4 \times 10^{2}$. The pressure drop is measured versus the flow rate at various fiber concentration $c$. For each $c$ we conduct a series of $\left(U_{b}\right.$, $d P / d x$ )-measurements.

Figure 1 shows the experimental data on the Prandtl-Kármán (PK)-coordinates: $\left(R e_{\tau}, U_{b}^{+}\right)$. The Newtonian data $(c=0)$ agree reasonably well with the PK law

$$
U_{b, P K}^{+}=\frac{1}{\kappa} \log R e_{\tau}+0.3,
$$

which is plotted in Fig. 1 with the solid line. Here, $\kappa \approx 0.4$ is the von Kármán constant. The fibre suspensions are drag reduced, with $U_{b}^{+}$exceeding Eq. (3). The video recordings reveal that in the drag reduction regime the suspensions consist of a rigid plug in the core, surrounded by a turbulent annulus. With increasing $R e_{\tau}$, the plug shrinks and eventually the plug disappears completely. During this process, the flow becomes Newtonian, which is seen in Fig. 1, as the data approach Eq. (3).

With decreasing $R e_{\tau}$, on the other hand, the plug grows, and eventually, when the plug diameter reaches the pipe diameter, the system clogs. To prevent clogging we conduct each measurement series from large to small $R e_{\tau}$ and stop when $U_{b}^{+}$drops below Eq. (3), which defines the clogging Reynolds number $R e_{\tau, c}$. Using that the fiber network yield stress $\tau_{c}$ is approximately equal to the wall shear stress at $R e_{\tau, c}$, and using Eqs. (1) and (2), we can express $\tau_{c}$ as follows:

$$
\tau_{c} \approx \rho\left(\frac{\nu R e_{\tau, c}}{D}\right)^{2}
$$




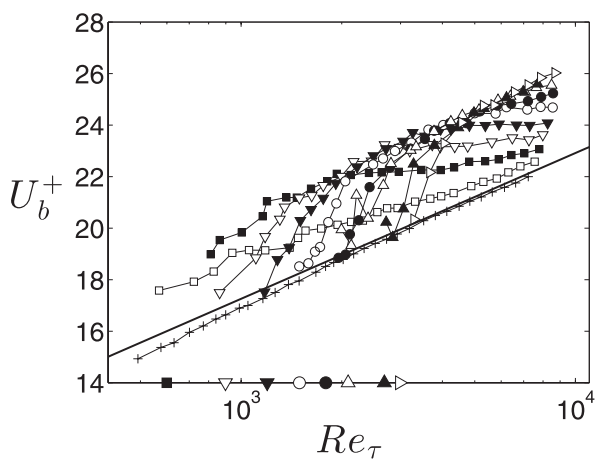

FIG. 1. Scaled bulk flow versus frictional Reynolds number in drag-reduced pipe flow using nylon fibers at several volume fractions $c:+, c=0 ; \square, c=0.72 \times 10^{-3} ; \mathbf{\square}, c=1.44 \times 10^{-3} ; \nabla, c=2.16 \times 10^{-3} ; \mathbf{\nabla}, c=2.88 \times 10^{-3} ; \bigcirc, c=3.60$ $\times 10^{-3} ; \bullet, c=4.31 \times 10^{-3} ; \Delta, c=5.03 \times 10^{-3} ; \mathbf{\Delta}, c=6.46 \times 10^{-3} ; \triangleright, c=7.17 \times 10^{-3}$. The solid line is the Prandtl-Kármán law (Eq. (3)). The markers on the abscissa indicate the clogging Reynolds number $R e_{\tau, c}$ for each fiber concentration.

The data in Fig. 1 indicate that the clogging Reynolds number $R e_{\tau, c}$ increases linearly with the fiber concentration $c$, which means that the network yield stress $\tau_{c}$ scales with the squared concentration. For our experiment, we find: $\tau_{c} \approx C_{2} c^{2}$, with $C_{2} \approx 7.2 \times 10^{4} \mathrm{~Pa}$. The $c^{2}$ dependence of $\tau_{c}$ reflects that $\tau_{c}$ is proportional to the number of physical contacts between the fibers.

To clearly demonstrate this quadratic scaling, we plot in Fig. 2, the excess bulk flow $\Delta U_{b}^{+}$ $=U_{b}^{+}-U_{b, P K}^{+}$as a function of $\tau_{w} / \tau_{c}=\rho R e_{\tau}^{2} v^{2} /\left(D^{2} C_{2} c^{2}\right)$. This figure essentially shows the fiberinduced flow changes with varying wall shear stress $\tau_{w}$. To demonstrate that clogging occurs at a $\tau_{w}$ that is proportional to $c^{2}$, we have collapsed the data from different $c$ on a single curve by scaling $\tau_{w}$ with $c^{2}$. The collapse further means that drag reduction in the plug-flow regime is fully determined by a single similarity variable $R e_{\tau} / c$. It is noted that the data for the smallest $c(\square)$ slightly deviate from the similarity curve, indicating that the plug-flow regime has not been fully reached for $c r_{a} \approx 0.3$.

We formulate a model to explain the drag reduction in the plug-flow regime. In this model, the flow consists of a rigid plug, surrounded by a Newtonian annulus. As sketched in Fig. 3(a) the surface of the plug is located at $r=r_{c}$, where the hydrodynamic shear stress is exactly balanced by the fiber network yield stress $\tau=\tau_{c}$. For $r<r_{c}$, the fiber network can support the hydrodynamic stress $\tau_{c}>\tau$, and the plug moves with a uniform velocity

$$
\frac{d U}{d r}=0 \text { if } r<r_{c}
$$

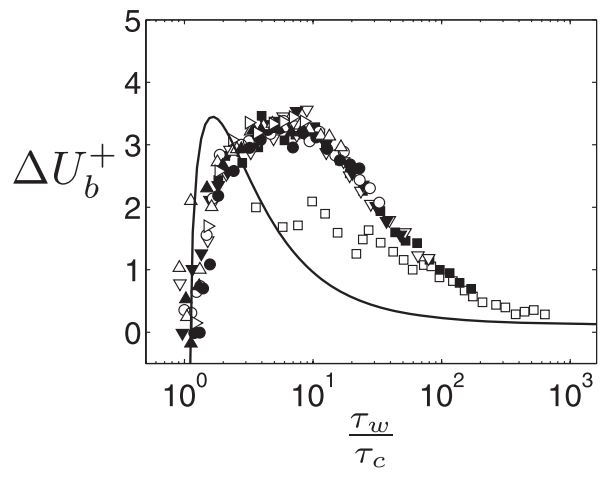

FIG. 2. Excess scaled bulk flow versus wall shear stress scaled with yield stress: $\tau_{w} / \tau_{c}=\rho R e_{\tau}^{2} v^{2} /\left(D^{2} C_{2} c^{2}\right)$. Markers are explained in Fig. 1. The solid line is our model (Eq. (5)) for $R e_{\tau, c}=10^{3}$. 

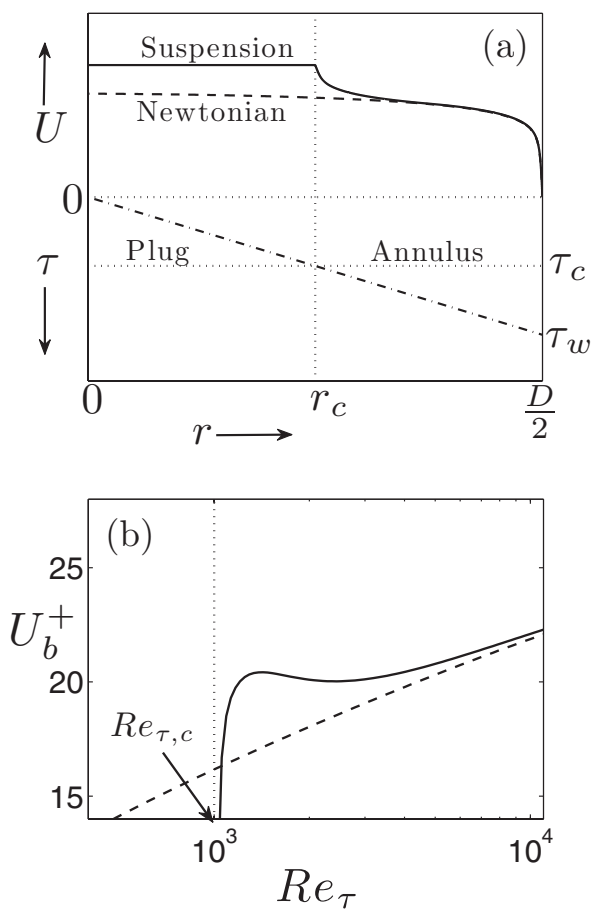

FIG. 3. Modeled flow using Eq. (5). (a) The upper half shows the modeled velocity $U$ as a function of the radial coordinate $r$, for Newtonian flow (dashed line) and drag reduced fiber suspension flow (solid line). The centreline and the pipe wall correspond to $r=0$ and $r=D / 2$, respectively. The model is based on dividing the cross section into a solid plug for $r<r_{c}$ and a Newtonian annulus for $r>r_{c}$. Here, $r_{c}$ corresponds to the point in the pipe where the shear stress $\tau$ equals the fiber network yield stress $\tau_{c}$. In the lower half, the dashed-dotted line shows the shear stress as a function of $r$. The wall shear stress is indicated with $\tau_{w}$. The figure illustrates that for $r<r_{c}$ the shear stress is smaller than the yield stress $\tau<\tau_{c}$, resulting in a solid plug, while for $r>r_{c}$ the shear stress exceeds the yield stress $\tau>\tau_{c}$, corresponding to the turbulent annulus. (b) The scaled bulk flow $U_{b}^{+}$versus the frictional Reynolds number $R e_{\tau}$. The solid line shows a fiber suspension with a clogging Reynolds number of $\operatorname{Re}_{\tau, c}=10^{3}$. The dashed line shows the Newtonian flow.

For $r>r_{c}$, on the other hand, the hydrodynamic stress disintegrates the fiber network $\tau>\tau_{c}$. The flow profile in this annulus is governed by the shear stress balance

$$
\mu \frac{d U}{d r}+\tau^{R}=-\frac{2 \tau_{w} r}{D} \text { if } r>r_{c} .
$$

Here, $\mu d U / d r$ is the viscous shear stress and $\tau^{R}$ is the Reynolds shear stress. The right hand side of Eq. (5b) is the total shear stress $\tau$ in the system, which by definition is a linear function of $r$. In Eq. (5b), we have neglected the stress produced by the fibers. This assumption is based on the experimental observation in Fig. 2, that for large $\tau_{w} / \tau_{c}$ the flow becomes Newtonian, which means that the fiber stress approaches zero. For not too large $\tau_{w} / \tau_{c}$ on the other hand, however, the fiber stress in the annulus is non-zero. This means that the turbulence in the annulus is non-Newtonian. We do not take these effects into account, since there are no models available for the stress induced by entangled fibers that are larger than the Kolmogorov length scale. Deriving such models is not the focus of this work. For simplicity, we therefore assume that the flow in the annulus is Newtonian. This does not mean that the flow profile is unchanged compared to the flow without fibers. As we will argue below, the presence of the central plug dampens the Reynolds stress in the annulus. As an effect the flow profile changes, and the friction factor reduces.

The Reynolds stress in Eq. (5b) is modeled using the mixing length model with the Van Driest damping ${ }^{12}$

$$
\tau^{R}=\left(\kappa r_{m}\left[1-e^{-\frac{r_{m} U_{\tau, l}}{260}}\right] \frac{d U}{d r}\right)^{2}
$$


Here, $r_{m}$ is the mixing length. In the annulus, the flow is bounded by the pipe wall and the plug surface. The latter is modeled as a flat no-slip surface at $r=r_{c}$ that is moving with the speed of the plug. The mixing length $r_{m}$ is therefore defined as the smallest distance to either the pipe wall or the plug surface

$$
r_{m}=\min \left(D / 2-r, r-r_{c}\right) .
$$

Accordingly, we base the local friction velocity $U_{\tau, l}$ in Eq. (5c) on the shear stress at the pipe wall $\tau_{w}$ or the shear stress at the plug surface $\tau_{c}$.

Figure 3(a) shows the velocity profile $U(r)$, modeled by Eq. (1), for $R e_{\tau}=2 \times 10^{3}$ and $R e_{\tau, c}$ $=10^{3}$. The suspension velocity is plotted with the solid line and the Newtonian velocity is plotted with the dashed line. It is noted that a similar profile is predicted by the $k-\epsilon$ model, applied to the water annulus in oil-water pipe flow. ${ }^{13}$ Drag reduction is reflected by the larger suspension velocity than the Newtonian velocity. As shown in Fig. 3(a), the larger velocity in the suspension is the effect of a sharp velocity increase in the annulus close to the plug surface. This increase is due to a reduction of the mixing length, due to the geometrical constraint imposed by the plug on the turbulent eddies.

The bulk flow $U_{b}$ is the weighted average of the velocity profile: $U_{b}=\frac{8}{D^{2}} \int_{0}^{D / 2} U(r) r d r$. In our model, the scaled bulk flow $U_{b}^{+}$is a function of the frictional Reynolds number $R e_{\tau}$ and the clogging Reynolds number $R e_{\tau, c}$. In Fig. 3(b), we plot our model results on the $\left(R e_{\tau}, U_{b}^{+}\right)$-plane using $R e_{\tau, c}$ $=10^{3}$. The model correctly reproduces the experimental trends. For $R e_{\tau}>R e_{\tau, c}$, the bulk flow exceeds the Newtonian value, which corresponds to drag reduction. When $R e_{\tau}$ is further increased, the drag reduction passes through a maximum and decreases asymptotically to zero, corresponding to Newtonian flow. In Fig. 2, we plot the model together with the experimental data. The model agrees qualitatively, but differs quantitatively, with a faster descent towards Newtonian flow.

Our model is based on the concept of a yield stress, needed to disintegrate the fiber network. When the wall shear stress is below the yield stress, the network spans the whole pipe cross section and the pipe is clogged. Above the yield stress, the network shrinks inwards, due to the growth of a turbulent annulus. In this regime, the flow is drag reduced, which we explain in this Letter by a two-layer model, with a flat profile in the core, surrounded by a Newtonian annulus. According to the model, the rigid fiber network in the core inhibits the size of the turbulent eddies in the annulus, effectively dampening the Reynolds stress, resulting in drag reduction. It is emphasized that this is a model picture and that in reality the drag reduction is probably accompanied with other changes in the turbulence structure.

While the model qualitatively explains the drag reduction mechanism in the plug-flow regime, there are some quantitative differences in the shape of the flow curve on the Prandtl-Kármán coordinates. These discrepancies are, at least partly, due to the neglect of a fiber stress term in Eq. (5b). As opposed to sub-Kolmogorov fibers, the form of this stress is not known for concentrated suspensions of fibers that are larger than the Kolmogorov length scale. However, the observation that for large Reynolds numbers the flow is Newtonian implies that the fiber stress is zero in this limit. This explains the success of our model to capture the qualitative trends.

We thank B. J. Boersma and R. Delfos for technical support and helpful discussions. The research has been partially supported through the PETROMAKS programme funded by the Research Council of Norway.

${ }^{1}$ P. S. Virk, “An elastic sublayer model for drag reduction by dilute solutions of linear macromolecules," J. Fluid Mech. 45, 417-440 (1971).

${ }^{2}$ H. J. Xu and C. K. Aidun, "Characteristics of fiber suspension flow in a rectangular channel," Int. J. Multiphase Flow 31, 318-336 (2005).

${ }^{3}$ N. K. Madavan, S. Deutsch, and C. L. Merkle, "Reduction of turbulent skin friction by microbubbles," Phys. Fluids 27(2), 356-363 (1984).

${ }^{4}$ M. D. Warholic, G. M. Schmidt, and T. J. Hanratty, "The influence of a drag-reducing surfactant on a turbulent velocity field," J. Fluid Mech. 388, 1-20 (1999).

${ }^{5}$ G. Gust, "Observations on turbulent-drag reduction in a dilute suspension of clay in sea-water," J. Fluid Mech. 75, 29-47 (1976).

${ }^{6}$ C. M. White and M. G. Mungal, "Mechanics and prediction of turbulent drag reduction with polymer additives," Ann. Rev. Fluid Mech. 40, 235-256 (2008). 
${ }^{7}$ J. J. J. Gillissen, B. J. Boersma, P. H. Mortensen, and H. I. Andersson, “Fibre-induced drag reduction,” J. Fluid Mech. 602, 209-218 (2008).

${ }^{8}$ J. J. J. Gillissen, "Polymer flexibility and turbulent drag reduction,” Phys. Rev. E 78(4), 046311 (2008).

${ }^{9}$ P. F. W. Lee and G. G. Duffy, "Relationships between velocity profiles and drag reduction in turbulent fiber suspension flow," AIChE J. 22, 750-753 (1976).

${ }^{10}$ J. J. J. Gillissen, B. J. Boersma, P. H. Mortensen, and H. I. Andersson, "The stress generated by non-Brownian fibres in turbulent channel flow simulations," Phys. Fluids 19, 115107 (2007).

${ }^{11}$ T.-Q. Li, J. D. Seymour, R. L. Powell, M. J. McCarthy, K. L. McCarthy, and L. Ödberg, "Visualization of flow patterns of cellulose fiber suspensions by NMR imaging," AIChE J. 40, 1408-1411 (1994).

${ }^{12}$ E. R. Van Driest, "On turbulent flow near a wall," J. Aero. Sci. 23, 1007-1011 (1956).

${ }^{13}$ A. Huang, C. Christodoulou, and D. D. Joseph, "Friction factor and holdup studies for lubricated pipelining II laminar and $k-\epsilon$ models of eccentric core flow," Int. J. Multiphase Flow 20, 481-491 (1994). 